\title{
Untersuchungen des Kohlenhydrat- und Fettstoffwechsels bei Prädiabetes*
}

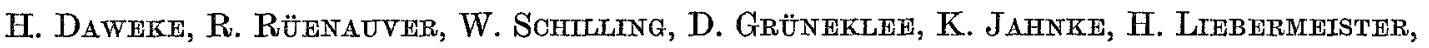 \\ F.A. GRIEs und K. ObERDISSE \\ 2. Medizinische Universitätsklinik und Diabetes-Forschungsinstitut, Universität Düsseldorf (Direktor: Prof. Dr. K. \\ OBERDISSE)
}

Eingegangen am 19. Februar 1968

\begin{abstract}
Investigations of Carbohydrate and Fat Metabolism in Pre-Diabetes

Summary. Because of the known changes of fat metabolites and elevated serum insulin levels in obesity, only investigations of normal-weight prediabetics can result in findings that are characteristic for genetically determined prediabetes. Serum insulin-Jike activity (ILA) and immunoreactive serum insulin (IRT) during oral GTT, free fatty acids (FFA) during i.v. GTT, and in some of the subjects cholesterol, triglycerides and esterified fatty acids were examined in 66 persons where both parents were diabetics and compared with a normal control group. Subjects with pathological oral and/or i.v. GTT and those more than $10 \%$ overweight were excluded and evaluated separately: - A significant elevation of fasting ILAlevels was observed only in the overweight group, but not in normal-weight prediabetics; fasting IRI-levels were rather low in all groups. In normal-weight prediabetics the rise in IRI and ILA during oral GTT was less than that in normal subjects and was delayed, a diminished insulin-reserve was found with both methods, and the decrease in FFA levels during i.v. GTT was significantly smaller. No other disturbance of fat metabolism was observed. - The following conclusions were reached: 1. Elovated fasting ILA levels are not the rule in normal. weight prediabetios and must be attributed to concomitant overweight or obesity. They cannot be considered characteristic of genetically determined prediabetes. 2. Since IRI is also not increased, there is no reason to assume insulinantagonistic mechanisms in the prediabetic state. - 3. The delayed and diminished rise of insulin after glucose which we found in good accordance with both methods for insulin-determination, as well as the diminished insulin-reserve and the delayed decrease of FFA after glucose, suggests an early and primary impairment of insulin secretion. - 4. Since investigations in nondiabetic and monozygotic twins of diabetics have confirmed the results described above, the possibility of detecting the prediabetic state in individual cases is conceivable.
\end{abstract}

Etude sur le métabolisme des hydrates de carbone et des lipides dans le prédiabète

Résumé. Les altérations du métabolisme lipidique et les taux élevés d'insuline chez les obèses étant bien connus, il convient de n'étudier les caractéristiques du prédiabète par transmission génétique que chez les prédiabétiques de poids normal. Chez 66 sujets avec deux parents diabétiques, nous avons étudié l'activité insulinosemblable (ILA) et l'insuline immunoréactive (IRI) sérique après surcharge

* Teilweise vorgetragen auf dem Second Annual Meeting European Association for the Study of Diabetes, 6. - 8. 7. 1966 in Aarhus, Dänemark, dem 72. KongreB der Deutschen Gesellschaft für Innere Medizin in Wiesbaden, April 1966 und dem 6. Kongreß der Internationa. Ien Diabetes Federation, 30. 7. - 4.8.1967 in Stockholm, Schweden. - Die Untersuchungen wurden mit dankenswerter Unterstützung der Deutschen Forschungsgemeinschaft und des Landesamtes für Forschung des Landes Nordrhein-Westfalen durchgeführt. glucidique par voie orale (o. GTT), les acides gras libres (FFA) après surcharge glucidique par voie intraveineuse (i.v. GTT) et dans quelques cas, le cholestérol, les triglycérides et los acides gras estérifiés (EFA) sériques. Ces mesures ont óté comparées à celles obtenues chez un groupe normal de contrôle. Les personnes avec un o.GTT et/ou un i.v. GTT pathologiques et celles avec un poids dépassant la normo de plus de $10 \%$ ont été évaluées séparément. - Alors que les prédiabétiques obèses présentaient une élévation des taux de l'ILA à jeun, ce n'était pas le cas pour les prédiabétiques de poids normal. Les taux de l'IRI à jeun étaient plutôt bas dans les deux groupes. Pour les prédiabétiques de poids normal l'élévation de l'IRT et de l'TLA pendant l'o.GTT était moindre et retardée, indiquant une réserve insulinique diminuée, et la dépression des FIF A pendant l'i.v. GTT éteit moins accusée. Nous n'avons pas constaté d'autre déviation du métabolisme lipidique. - Nous sommes arrivés aux conclusions suivantes: - 1. Des taux élevés d'ILA no sont pas fréquents chez les prédiabétiques do poids normal. Les taux élevés sont en général à attribuer à une obésité concomitante. Ils ne sont pas caractéristiques du prédiabète par transmission génétique. - 2. Les taux d'IRI n'étant pas élevés, il n'y a pas de raison d'admettre la présence d'un antagoniste à l'insuline pendant la phase prédiabétique. - 3. L'élévation moindre et retardée de l'insuline après glucose que nous avons notée en bonne concordance avec les deux méthodes de mesure d'insuline, aussi bien que la réserve insulinique diminuée et la dépression retardée des FFA après glucose suggèrent une perturbation précoce et primaire de la sécrétion insulinique. - 4. Les études de jumeaux monozygotes non-diabétiques de diabétiques ayant confirmé les résultats décrits ci-dessus, la possibilité de dépister la phase prédiabétique semble désormais donnée.

Zusammenfassung. Wegen der bekannten Veränderungen der Metabolite des Fettstoffwechsels und der erhöhten Seruminsulinwerte bei Adipositas können nur Untersuchungen normgewichtiger Prädiabetiker charakteristische Befunde für den genetisch determinierten Prädiabetes erbringen. Bei 66 Nachkommen zweier manifest diabetischer Elternteile wurden die insulinähnliche Aktivität im Serum (ILA) und das immunologisch erfaßbare Seruminsulin (IRI) im oralen GTT, die freien Fettsäuren (FFS) im i.v. GTT und bei einem Teil Cholesterin, Triglyceride und Esterfettsäuren untersucht und mit einem Normalkollektiv verglichen. - Probanden mit pathologischen oralen und/oder i.v. GTT und mehr als $10 \%$ Übergewichtige wurden ausgesondert und getrennt bewertet. - Signifikant erhöhte ILA-Nüchternwerte ließen sich nur bei der Gruppe der übergewichtigen, nicht der normgewichtigen Prädiabetiker nachweisen, die Nüchternwerte des IRI lagen in allen Gruppen eher niedrig. Bei normgewichtigen Prädiabetikern war der Anstieg von IRI und ILA im or. GTT geringer und verzögert, die Insulinreserve bei beiden Meßmethoden signifikant vermindert, der Abfall der FFS im i.v. GTT signifikant geringer. Darüberhinaus bestanden keino nachweisbaren Fettstoffwechselstörungen. - Folgende Schlußfolgerungen wurden gezogen: - 1. Erhöhte ILA-Nüchternwerte 
sind bei normgewichtigen Prädiabetikern nicht die Regel und sind auf eine gleichzeitig bestehende Übergewichtigkeit bzw. Fettsucht zurückzuführen. Sie können nicht als charakteristisch für den genetisch determinierten Prä. diabetes angesehen werden. - 2. Da auch das IRI nicht orhöht ist, besteht keine Veranlassung, insulinantagonistische Mechanismen im Stadium des Prädiabetes anzunehmen. - 3. Der mit beiden Insulinbestimmungsmethoden übereinstimmend gefundene verzögerte und verringerte Anstieg des Insulins nach Glucosegabe, die verringerte Insulinreserve und der verzögerte Abfall der FFS nach Glucosegabe lassen vielmehr eine frühe und primäre Störung der Insulinsekretion vermuten. - 4. Da die Untersuchungen an nichtdiabetischen monozygoten Zwillingen diabetischer Partner die geschilderten Ergebnisse bestätigen, erscheint die biochemische Erfaßbarkeit der prädiabetischen Phase auch im Einzelfall denkbar.

Key-words. Prediabetes, genetically determined prediabetes, carbohydrate metabolism in genetically determined prediabetes, fat metabolism in genetically determined prediabetes, serum insulin in genetically determined prediabetes, insuline reserve in genetically determined prediabetes, twins of diabetics.
Die Diagnose Prädiabetes oder potentieller Diabetes ist immer eine retrospektive. Der Begriff schließt nachweisbare Störungen des Kohlenhydratstoffwechsels aus. Für Stoffwechseluntersuchungen ist man darauf angewiesen, Personen mit dem höchsten Erkrankungsrisiko für Diabetes zu untersuchen. Unabhängig vom noch immer umstrittenen Vererbungsmodus kann man annehmen, daß Abkömmlinge von Eltern, die beide diabetisch sind, sogenannte genetisch determinierte Prädiabetiker, besonders häufig einen Diabetes mellitus entwickeln werden und somit das Stadium des Prädiabetes durchlaufen müssen.

Aus einer bei solchen Personen bereits 1961 von STEINTE et al. [34] und später auch von anderen Autoren [3, 35, 8, 22] festgestellten Erhöhung der Nüchternwerte der insulinähnlichen Aktivität (ILA) wurde geschlossen, daß der Diabetesmanifestation eine Phase verminderter peripherer Insulinempfindlichkeit oder eine Hemmung bzw. Inaktivierung des Insulins vorausgehe. Diese Anschauung wurde fast allgemein anerkannt. Mit immunologischer Meßtechnik fanden sich die Insulinnüchternwerte jedoch im Normbereich $[3,12,26,6,33]$. Bei der Erfassung des Funktionsablaufes der Insulinfreisetzung nach Glucosegabe waren die Ergebnisse sehr unterschiedlich: mit biologischer Methodik ließ sich ein verzögertes und verringertes [35, 3] bzw. verringertes [8] Ansteigen, mit immunologischer Methodik dagegen ein normaler Insulinanstieg mit verzögertem Abfall [3], keine Ab. weichung von der Norm [12,33], ein stärkeres [26] und schließlich ein verzögertes und verringertes Ansteigen [6] nachweisen.

Zum Teil lassen sich diese Widersprüche durch den Einfluß der Adipositas erklären, die entsprechend ihrem Ausmaß zu einer Erhöhung der Seruminsulinkonzentration führen kann $[25,9,2,29,16,17,21]$, was zur Zeit der ersten Untersuchungen von Prädiabetikern nicht bekannt war $[34,3,35]$. Die Aussagekraft der ILA-Befunde ist außerdem wegen der großen Streuung der Methode, der großen individuellen Schwankungen der Meßwerte und der Unspezifität der Methode begrenzt. Schließlich ist bei dem nicht gesicherten Erbgang des Diabetes das 100\%ige Vorliegen eines Prädiabetes bei Kindern diabetischer Eltern durchaus zweifelhaft, weswegen statistische Vergleiche kleiner Kollektive $[35,12,33,16,6] \mathrm{mit}$ einem großen Unsicherheitsfaktor belastet sind.
Zur Klärung der Frage, ob im Stadium des Prädiabetes unabhängig von einer Adipositas biochemische Veränderungen nachweisbar sind, und zur Klärung der unterschiedlichen vorliegenden Befunde haben wir bei einer größeren Zahl genetisch determinierter Prädiabetiker und Normalpersonen vergleichend das Seruminsulin sowohl mit der biologischen Methode zur Bestimmung der ILAA am Fettgewebe als auch mit immunologischer Technik im oralen Glucosetoleranztest (or. GTT) untersucht. Außerdem wurden die freien Fettsäuren (FFS) im intravenösen Glucosetoleranztest (i. v. GTT) und bei einem Teil Cholesterin, Triglyceride und Esterfettsäuren im Serum bestimmt.

\section{Patientengut und Methodik}

66 nichtdiabetische Nachkommen zweier diabetischer Eltern ( $35 \pm$ 12.2 Jahre, Gewicht: BRocA-index $117 \pm 25.4 \%$ und 58 Normalpersonen ohne familiäre diabetische Belastung (Alter $31 \pm 9.3$ Jahre, Gewicht: BrocA-index $96 \pm 12.2 \%$ ) wurden nüchtern stationär und in einigen Fällen auch ambulant mit dem oralen Glucosetoleranztest (or. GTT) und zum größten Teil mit dem intravenösen Glucosetoleranztest (i.v. GTT) untersucht. Auf eine vorbereitende Diät wurde nach den Untersuchungen von WIHKERSON et al. [15] verzichtet.

Vor und während des or. GTT mit einer Standarddosis von $100.0 \mathrm{~g}$ Glucose, in $250 \mathrm{ml}$ Wasser gelöst, wurde gleichzeitig mit der Bestimmung der Blutzuckerkonzentration zu den Zeiten $0,30,60,120$ und 180 Min. Blut aus der Cubitalvene zur Bestimmung der Seruminsulinkonzentration entnommen. Nach Absetzen des Blutkuchens und Abkühlen auf Zimmertemperatur wurde das Blut zentrifugiert und das gewonnene Serum bei $-22^{\circ} \mathrm{C}$ aufbewahrt. 12 Std. vor der Seruminsulinbestimmung wurde es in eine Temperatur von $+4^{\circ} \mathrm{C}$ gebracht und langsam aufgetaut.

Nach einer bis zwei Wochen wurde beim größten Teil der Personen der i.v. GTT mit $25.0 \mathrm{~g}$ Glucose (50 $\mathrm{ml}$ einer 50\%igen Glucoselösung in 2-4 Min. i.v.) durchgeführt, nachdem die Probanden nüchtern eine halbe Stunde ruhig liegend verbracht hatten. Aus der ungestauten Cubitalvene wurde unter Vermeidung jeder Muskelmassage Blut zur Bestimmung von Serumfettwerten zu den Zeiten der gleichzeitig durchgeführten Blutzuckerbestimmung, nämlich nach 0,10 , 
20, 40 und 60 Min., entnommen, in vorgekühlte Zentrifugenröhrchen gegeben und bis zur Aufarbeitung bei $+4^{\circ} \mathrm{C}$ gehalten. Die Fettanalysen erfolgten innerhalb von 2 Std. nach der Blutabnahme. Die Bestimmung der freien Fettsäuren (FFS) konnte bei 56 genetisch determinierten Prädiabetikern durchgeführt werden.

Die Bestimmung der Blutzuckerkonzentration wurde mit dem Autoanalyser ,,Technikon“ in Doppelbestimmungen nach HoFHMAN [14] und GRADY und Lamar [11] vorgenommen. Die Bestimmung der insulinähnlichen Aktivität (ILA) erfolgte sechsfach bei einer Serumverdünnung von 1:2 mit der modifizierten $[8,9]$ Methode der Oxydation von Glucose-1 $-{ }^{14} \mathrm{C}$ zu ${ }^{14} \mathrm{CO}_{2}$ am epididymalen Fettgewebe der Ratte nach MarTin et al. [20], die immunologische Insulinbestimmung (IRI) mit der radio-immunologischen Methode C nach HALES und RANDLE [13] unter generellem Zusatz von EDTA zum Reaktionsmedium [28]. Die FFS wurden titrimetrisch modifiziert nach DoLe [10, 19, 36] und bei einem Teil der Probanden Cholesterin mit dem Autoanalyser nach ZLATKIs et al. [40], Triglyeeride enzymatisch nach KREUTz [18] und Esterfettsäuren (EFS) nach Roswnthal et al. [27] untersucht.

Den or. GTT sehen wir nach unseren Erfahrungen [30] dann als pathologisch an, wenn mindestens 3 von $6 \mathrm{Me}$ Mwerten oberhalb der ermittelten oberen biologischen Streuungsgrenze (Mittelwert des Normalkollektivs $+2 S_{D}$ ) liegen, also nüchtern $>90 \mathrm{mg} \%$, $30 \mathrm{Min} .>175 \mathrm{mg} \%, 60 \mathrm{Min} .>160 \mathrm{mg} \%, 90 \mathrm{Min}$. $>140 \mathrm{mg} \%, 120 \mathrm{Min} .>135 \mathrm{mg} \%, 180 \mathrm{Min} .>$ $105 \mathrm{mg} \%$. Beim i.v. GTT ist eine Konstante nach CoNARD [7] unter 1.40 (Mittelwert des Normalkollektivs $-2 S_{D}$ ) als pathologisch anzusehen [30]. Patientem mit manifestem Diabetes, d.h. dauernd erhöhtem Nüchternblutzucker und sporadischer Glucosurie wurden ausgesondert. Alle Probanden, die beim or. GTT und/oder i.v. GTT die angegebenen Grenzen überschrititen, wurden als latente Diabetiker bezeichnet. Der Index der Insulinreserve wurde modifiziert nach Sectzer und Surth [31] nach der Formel $\frac{\Delta \mathrm{ILA}}{\triangle \mathrm{BZ}}$ bzw. $\frac{\Delta \mathrm{IRI}}{\triangle \mathrm{BZ}}$ (nüchtern und $30 \mathrm{Min}$. oder 60 Min.) berechnet.

\section{Ergebnisse}

Wie in Tabelle 1 zusammengestellt, zeigten von den als Gruppe 1 bezeichneten 66 untersuchten Nachkommen zweier diabetischer Eltern 21 einen pathologischen i.v. - und/oder or. GTT, waren also latente Diabetiker (Gruppe 3), 45 hatten keine nachweisbare Kohlenhydratstoffwechselstörung und waren somit als Prädiabetiker zu bezeichnen (Gruppe 2). Von diesen waren 23 norm- bzw. untergewichtig (Gruppe 4), 22 hatten ein tbergewicht von mehr als $10 \%$ nach Broca, im Mittel $27 \pm 16,9 \%$ (Gruppe 5). Wie weiter aus der Tabelle ersichtlich, stimmen mittleres Alter

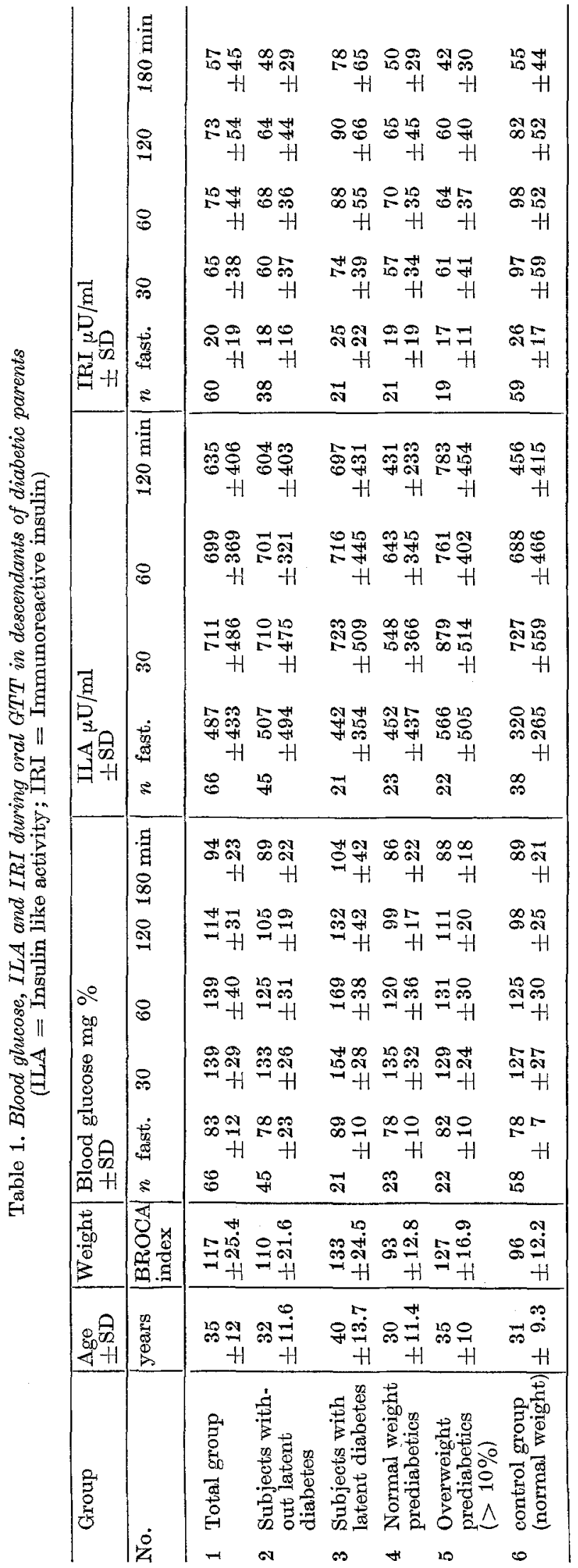


und Alterszusammensetzung der Gruppen der Prädiabetiker und Normalpersonen weitgehend überein, und auch nach Aufteilung der Prädiabetiker in Normalgewichtige und Übergewichtige ergeben sich durchaus vergleichbare Altersverhältnisse.
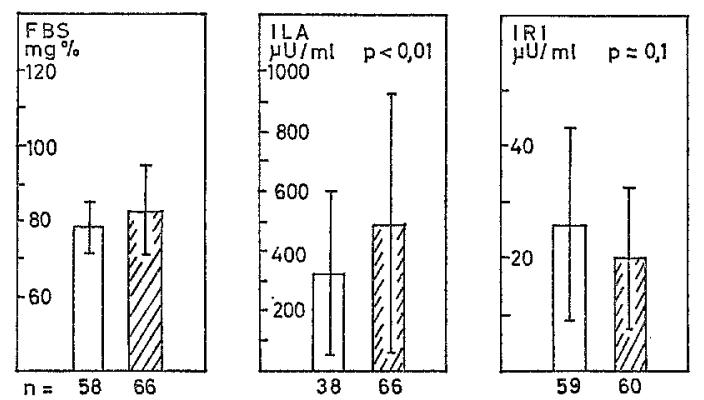

Serum fasting values of glucose, ILA and IRI in genetically determined prediabetics (both parents diabetic)
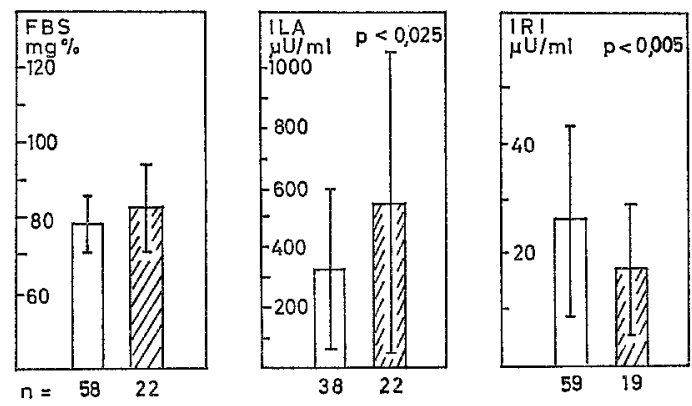

overweight $(>10 \%)$ both GTT normal

normal weight controls
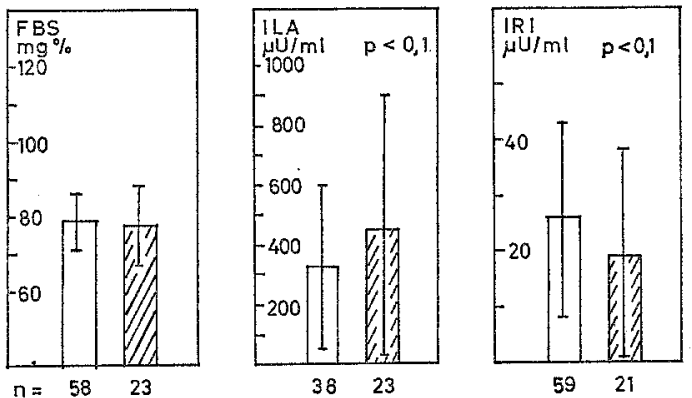

normal weight both GTT normal

normal weight controls

Fig. 1. Serum fasting values of glucose, ILA and IRI in genetically determined prediabetics (both parents diabetic). ILA fasting values are significantly elevated only in overweight not in normal weight prediabetics. $(\mathrm{FBS}=$ fasting blood sugar; ILA $=$ insulin-like activity; IRI = Immunoreactive insulin)

Abb. 1 zeigt die Nüchternwerte für Blutzucker, ILA and IRI der genetisch determinierten Prädiabetiker im Vergleich mit den entsprechenden Werten der Normalpersonen (s. Tabelle 1, Gruppen 1 und 6). Bei der Gesamtgruppe 1 einschlieBlich der latenten Diabetiker ist die ILA erhöht. Trotz der großen Streuung ist der Unterschied statistisch gesichert $(p<0.01)$. Das IRI ist eher niedriger als die Norm. Nach Aussonderung der Personen mit latentem Diabetes (pathologische GTT) sind die Prädiabetiker in übergewichtige und normalgewichtige Personen (Gruppen 5 und 4) unterteilt. Die 22 übergewichtigen Personen ohne
Glucosetoleranzstörung haben erwartungsgemäß im Mittel einen statistisch gesichert erhöhten ILA-Nüchrnwert (566 zu $320 \mu \mathrm{E} / \mathrm{ml} ; p<0.025)$. Das IRI liegt niedrig (17 zu $26 \mu \mathrm{E} / \mathrm{ml})$. Die normgewichtigen Prädiabetiker zeigen keine signifikante Erhöhung der ILA!

Das Gesamtkollektiv der genetisch determinierten Prädiabetiker (Gruppe 1) weist im or. GTT entsprechend dem Anteil von 21 latenten Diabetikern (zum Teil signifikant) erhöhte BZ-Werte auf (Tabelle 1). Für die ILA fällt ein geringerer Anstieg nach $30 \mathrm{Min}$. und ein verzögerter Abfall nach 120 Min. auf, während das IRI einen verringerten und verzögerten Anstieg mit Annäherung an die Norm erst nach 180 Min. zeigt. Nach 30 und 60 Min. finden sich statistisch erniedrigte Werte $(p<0.0005$ und $p<0.005$ ).

Bei der Aufteilung der Probanden in latente Diabetiker und Prädiabetiker (Abb. 2, Tabelle 1, Gruppen 3 und 2) sind die Blutzuckerwerte der latenten Diabetiker im or. GTT definitionsgemäß erhöht. Die Nüchtern-ILA der Prädiabetiker und die ILA der latenten Diabetiker, 120 Min. nach Glucosebelastung, sind signifikant erhöht, während alle anderen ILA-Werte im Streubereich der Norm liegen. Dasimmunologisch reagierende Insulin (IRI) zeigt im or. GTT vor allem bei den Prädiabetikern einen verringerten und verzögerten Anstieg. Signifikante Unterschiede gegen die Norm bestehen trotz gleicher BZ-Werte nach 30, 60 und 120 Min. Das IRI der 21 latenten Diabetiker steigt im Mittel höher an und nähert sich dem Normbereich.

Um den Einfluß der Adipositas auf die Höhe der Insulinwerte bei Prädiabetikern zu untersuchen, erfolgte eine Aufteilung der Probanden ohne pathologische Glucosetoleranz (Gruppe 2) in normgewichtige und übergewichtige (mehr als $10 \%$ Übergewicht nach Broda, Abb. 3, Tab. 1, Gruppen 4 und 5). Alle ILA-Werte der normgewichtigen Prädiabetiker liegen im Streubereich der Norm, während sich bei den übergewichtigen Prädiabetikern signifikante Unterschiede ergeben. Außer dem erhöhten ILA-Nüchternwert zeigt sich ein verzögerter Abfall der ILA der Fettsüchtigen. Für die normgewichtigen Prädiabetiker fällt ein verzögerter Anstieg der ILA auf, der von der Norm statistisch gesichertunterschieden ist. Die Insulinreserve $\frac{\Delta \mathrm{ILA}}{\triangle \mathrm{BZ}}$ beträgt nach 30 Min. $31 \%$ der Norm ( $p<0.0125)$, nach 60 Min. $47 \%$ der Norm $(p<0.05)$.

Das IRI steigt nicht nur bei den Normgewichtigen, sondern hier auch bei den Übergewichtigen verringert und verzögert an, wobei sich signifikante Unterschiede 
gegenüber der Norm nach 30 und 60 Min. ergeben. Die Insulinreserve des IRI der normgewichtigen Prädiabetiker beträgt nach $30 \mathrm{Min} .39 \%$ der Norm, ist also hoch signifikant erniedrigt $(p<0.0005)$, ebenso nach 60 Vin. mit $52 \%(p<0.0025)$.

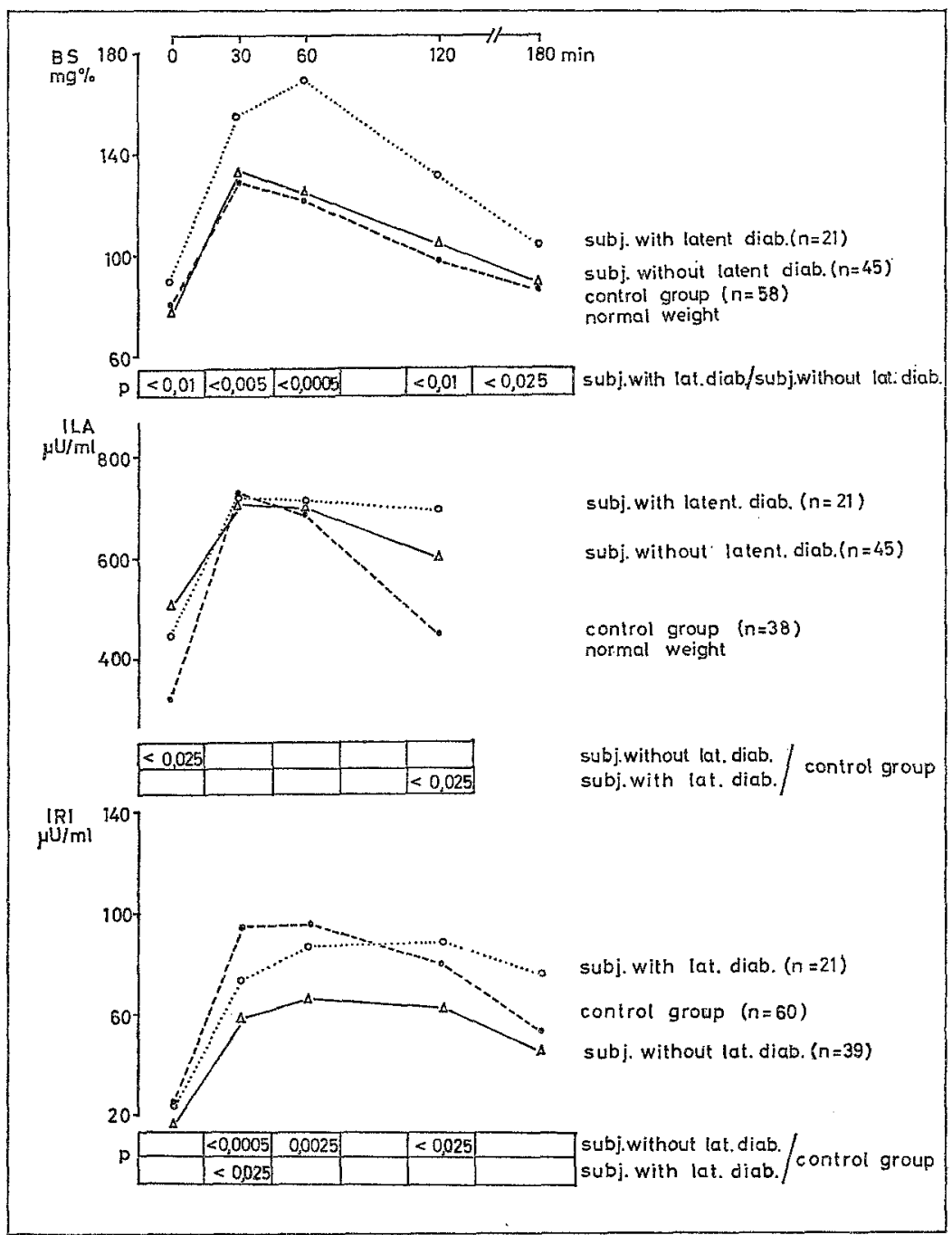

Fig. 2. ILA and IRI after oral GTT ( $100.0 \mathrm{~g}$ glucose) in descendants of diabetic parents with and without latent diabetes. Diminished and delayed increase of IRI particularly in prediabetics. (ILA = insulin-liko activity, IRI $=$ immunoreactive insulin; GTT = glucose tolerance test)

Im i.v. GTT zeigen die Personen mit latentem Diabetes eine signifikante Erhöhung der FFS-Nüchternwerte und einen gegenüber der Norm signifikant verminderten prozentualen Abfall (Tabelle 2 und Abb. 4). Bei der gleichen Aufteilung in norm- und übergewichtige Prädiabetiker ist der FFS-Nüchternwert nicht signifikant erhöht, doch findet sich ein gegenüber der Norm statistisch gesichert verminderter Abfall nach Glucose $(p<0.025)$. Während die Serumcholesterinspiegel keine Unterschiede erkennen lassen, sind die Esterfettsäuren (EFS) lediglich bei der Fettsucht erhöht. Für die Triglyceride sind die Endgruppen wegen der notwendigen Trennung in Geschlechter bei der statistischen Auswertung noch zu klein.

\section{Besprechung der Ergebnisse und Schlupfolgerungen}

Unsere Untersuchungen der ILA haben gezeigt, daß erhöhte Nüchternwerte bei normgewichtigen Prädiabetikern nicht die Regel sind. Sie sind vielmehr auf eine gleichzeitig bestehende Übergewichtigkeit bzw. Fettsucht zurückzuführen, bei der nach früheren Untersuchungen $[9,2$, 29] erhöhte Serumspiegel der ILA gefunden werden. So können erhöhte ILA-Nüchternwerte nicht für den genetisch determinierten Prädiabetes als charakteristisch angesehen werden.

Das immunologisch bestimmte Insulin fanden wir im Nüchternzustand bei den genetisch determinierten Prädiabetikern in Übereinstimmung mit anderen Autoren [3, 12, $26,6,33,37]$ nicht erhöht, sondern sogar erniedrigt. Es zeigte auch im Mittel keine Abhängigkeit von einer gleichzeitig bestehenden Ưbergewichtigkeit von $27 \%$ wie das bei Fettsüchtigen vergleichbaren Übergewichtsgrades auch von YALOW et al. [39] gezeigt wurde. Erst bei stärkerem Ửbergewicht von mehr als $70 \%$ und mehr als $138 \%$ fanden Kreisberg et al. [17] and PerLey et al. [23] das IRI auch nüchtern erhöht, und auch bei eigenen bisher unveröffentlichten Untersuchungen an 44 Adipösen mit normalem or. and i. v. GTT war die Erhöhung des Nüchtern-IRI bei Gruppen mit einem mittleren Übergewicht von $40 \%$ und $60 \%$ im Gegensatz zu der Gruppe mit einem Übergewicht von $102 \%$ nicht statistisch gesichert. Bei Mituntersuchung auch höherer Grade von Fettsucht ließ sich sogar eine direkte Korrelation von Nüchtern-IRI und Übergewicht erkennen (BAGDADE et al. [1]).

Nach unseren. Untersuchungen einschließlich der normalen FFS-Nüchternspiegel der Prädiabetiker besteht keine Veranlassung, insulinantagonistische Mechanismen im Stadium des Prädiabetes anzunehmen.

Bei der Aufteilung der untersuchten Probanden in Prädiabetiker und latente Diabetiker fällt die signifikante Erhöhung der Nüchtern-ILA bei den Prädiabetikern und der ILA 120 Min. nach Glucosegabe bei 
den latenten Diabetikern auf (Abb. 2). Beide Befunde lassen sich auf den hohen Anteil von übergewichtigen Probanden an diesen Gruppen zurückführen, 22 von 45 Probanden der Prädiabetiker (Abb. 3 und Tabelle 1) und 17 von 21 latenten Diabetikern mit einem

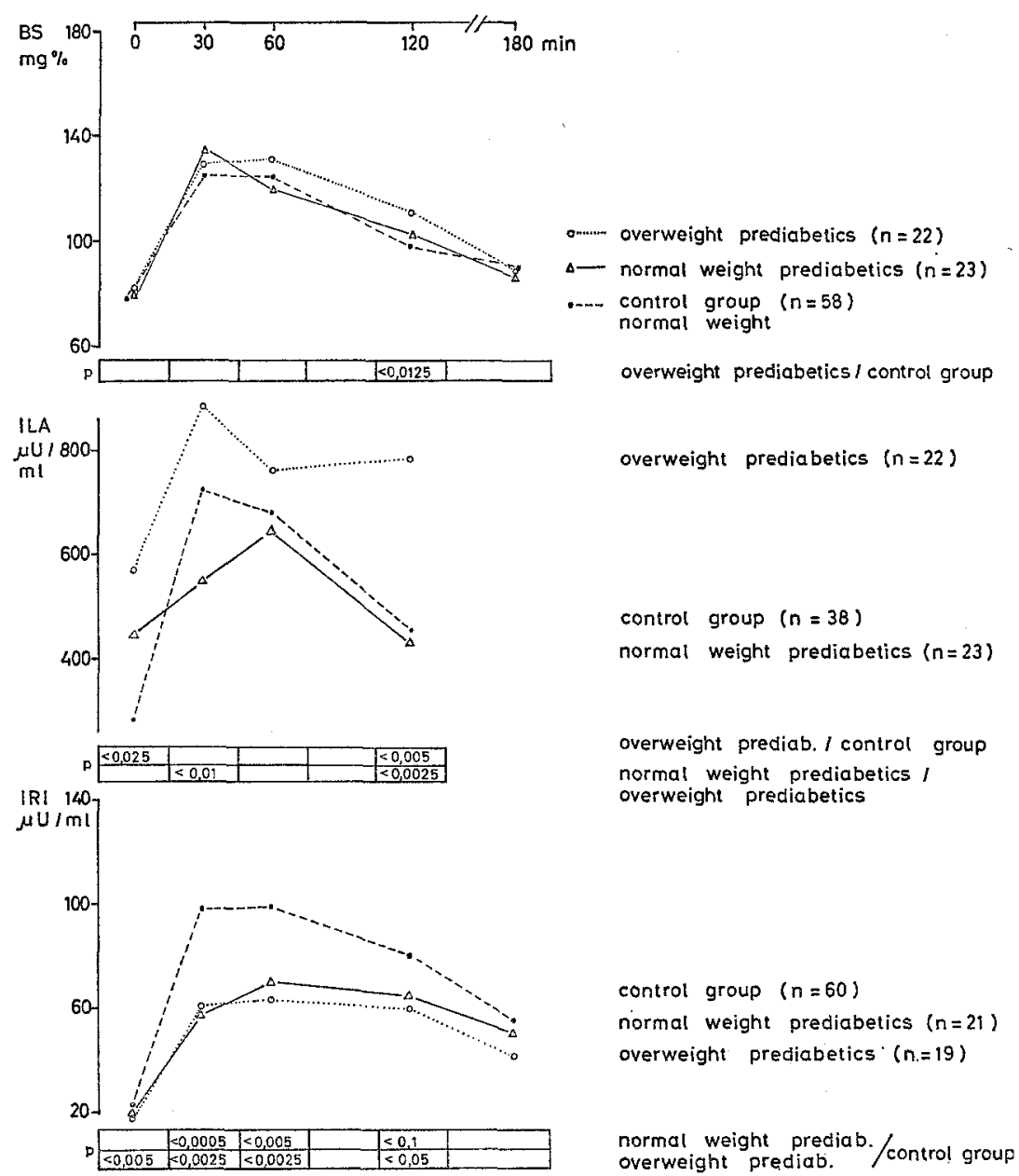

Fig. 3. ILA and IRI after oral GTT (100.0 $\mathrm{g}$ glucose) in genetically determined prediabetics normal or overweight $(>10 \%)$. Delayed and diminished increase of ILA and IRI in normal weight prediabetics. (ILA = insulin-like activity, IRI = immunoreactive insulin; GTT = glucose

mittleren Übergewicht von $40 \%$. Auch bei der Beurteilung des höheren Anstiegs des IRI nach Glucosebelastung bei der Gruppe von latenten Diabetikern gegenüber der von Prädiabetikern muß neben dem erhöhten Blutzuckerspiegel der Einfluß dieser relativ erheblichen Fettsucht berücksichtigt werden.

Der mit beiden Insulinbestimmungsmethoden übereinstimmend gefundene verzögerte und verringerte Anstieg des Insulins nach Glucosegabe bei normgewichtigen Prädiabetikern, und die verringerte Insulinreserve, läßt an eine frühe und primäre Störung der Insulinsekretion denken. Trotz dieser Abnormität ist die Glucosetoleranz noch normal. Dieser Befund bestätigt frühere mit biologischer Methodik durchge- führte Untersuchungen [35, 3, 8, 22], wobei allerdings nur wir selbst den Einfluß der Adipositas berücksichtigten. Mit immunologischer Technik wurden unsere Ergebnisse kürzlich von CoLwELL et al. [6] und Sнтма et al. [32] bestätigt, während RickexT's et al. [26] über einen höheren Insulinanstieg nach Glucose berichteten. Bei Berechnung der Gesamtmenge des ausgeschütteten Insulins im Verhältnis zum Blutzuckerspiegel fanden die Autoren jedoch auch hier eine im Verhältnis zur Norm verringerte Insulin selzretion auf Glucosereiz. Die Frage, warum trotz einer Insulinsekretionsstörung noch keine Störung der Glucosetoleranz auftritt, erklärt sich möglicherweise durch eine normalerweise überschüssige Insulinfreisetzung und die bekannte Insulinbindung in der Leber, die bei Prädiabetikern regulativ herabgesetzt sein könnte.

Die von uns gefundene verminderte Insulinreserve bei übergewichtigen Prädiabetikern müßte mit der eines Kollektivs übergewichtiger Normalpersonen gleicher Gewichtsklasse verglichen werden. Bei letzteren finden sich excessiv hohe Insulinspiegel nach Belastung mit Glucose $[16,17,21,23]$. Der statistische Unterschied der Insulinreserve zwischen diesen Gruppen würde dann noch größer. Auffallend ist das unterschiedliche Verhalten von IRI und ILA bei den übergewichtigen Prädiabetikern im Gegensatz zu dem bei normgewichtigen Prädiabetikern. Nach bisher unveröffentlichten Versuchen führt die Fettsucht bei geringerem Ausmaß nicht eher zur Erhöhung der ILA als des IRI.

Die Annahme einer frïhen und primären Insulinsekretionsstörung wird durch unseren Befund des signifikant verzögerten Abfalls der FFS im i.v. GTT unterstützt. In Übereinstimmung mit RICKETTS et al. [26] ließ sich für normgewichtige Prädiabetiker keine Erhöhung der FFS-Nüchternwerte nachweisen, wie sie von anderen Autoren [3] berichtet worden ist, die jedoch wie wir den verzögerten Abfall nach intravenöser Glucosegabe sahen.

Diese von uns gefundenen Veränderungen sind statistisch erfaßt, aber wegen mangelnder Kenntnis über den Vererbungsmodus des Diabetes mit einem Unsicherheitsfaktor belastet. Selbst wenn man bei Nachkommen zweier diabetischer Eltern in $100 \%$ das Vorliegen eines Prädiabetes akzeptiert, kann bei langsamer Entwicklung einer Insulinsekretionsstörung 
nur ein Teil der untersuchten Prädiabetiker solche Störungen aufweisen.

Die Annahme, daß die verzögerte und verringerte Insulinsekretion und der verzögerte Abfall der FFS
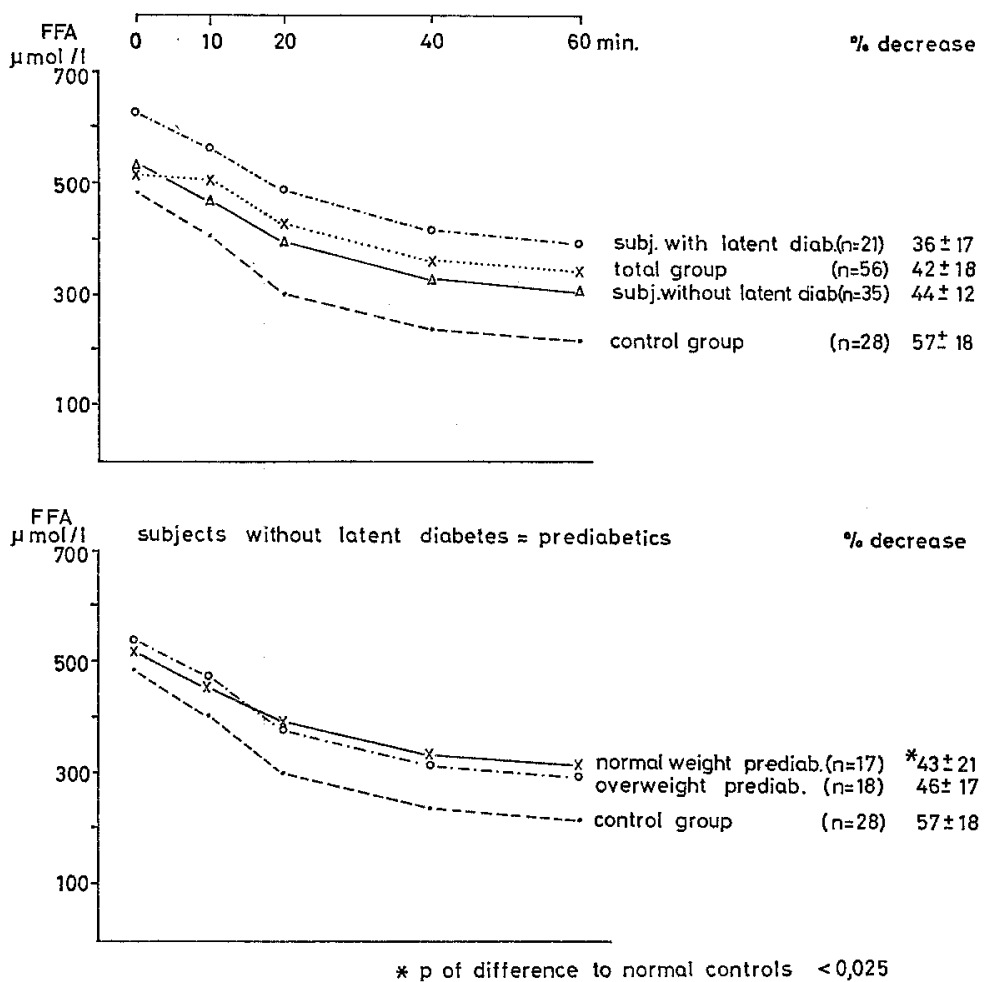

tatsächlich für die Phase des Prädiabetes charakteristisch sind, wird durch die Befunde bei nichtdiabetischen eineiigen Zwillingen von Diabetikern gestützt. 1966 berichteten wir über einen solchen Patienten [15], bei dem wir seit Jahren mehrfach den gleichen verzögerten und verringerten Anstieg des IRI, einen ILA-Abfall wie bei jugendlichen Diabetikern und einen verminderten FFS-Abfall nach Glucosegabe sahen. Eine Progredienz dieser abnormen Reaktionen ließ sich bisher nicht feststellen. In neuester Zeit konnte für zwei Gruppen von 6 nichtdiabetischen monozygoten $Z$ willingen, deren Zwillingspartner bereits diabetisch waren, mit immunologischer Technik diese verzögerte und verringerte Insulinsekretion nach Glucosereiz bestätigt werden (Cerasi et al. $[4,5]$ und Pyke et al. [24]).

Sollten sich diese Befunde bestätigen, bestände die Möglichkeit, die prädiabetische Phase nicht nur statistisch bei einem gefährdeten Personenkreis, sondern auch im Einzelfall mit den geschilderten Methoden zu erfassen.

Fig. 4. FFA during i.v. GTT (25.0 g glucose) in descendants of diabetic parents. Diminished decrease of FFA in normal weight prediabetics. $\left(\mathrm{FFA}=\right.$ free fatty acids, $\mathrm{GT}^{\mathrm{T} T}=$ glucose tolerance test)

Table 2. Serum lipids during i.v. GTT in descendants of diabetic parents

(FFA = free fatty acids; EFA = esterified fatty acids)

\begin{tabular}{|c|c|c|c|c|c|c|c|c|c|c|c|c|}
\hline & coup & Age & Weight & FFA $\mu \mathrm{m}$ & $\mathrm{ol} / \mathrm{L} \pm$ & SD & & & & choles- & trigly- & EFA \\
\hline & & years & BROCA & n fast. & 10 & 20 & 40 & $60 \mathrm{~min}$ & $\%$ & $\mathrm{mg} \%$ & $\mathrm{mmol} / \mathrm{l}$ & $\mathrm{mmol} / \mathrm{l}$ \\
\hline & Total group & $\begin{array}{r}37 \\
\pm 13\end{array}$ & $\begin{array}{r}117 \\
\pm \quad 26\end{array}$ & $\begin{array}{r}56 \\
558 \\
\pm 170\end{array}$ & $\begin{array}{r}502 \\
\pm 164\end{array}$ & $\begin{array}{r}425 \\
+163\end{array}$ & $\begin{array}{r}360 \\
\pm 156\end{array}$ & $\begin{array}{r}337 \\
\pm 164\end{array}$ & $\begin{array}{r}42 \\
\pm 18\end{array}$ & $\begin{array}{r}246 \\
\pm 68 \\
(29)\end{array}$ & $\begin{array}{l}1.2 \\
\pm 1 \\
(16)\end{array}$ & $\begin{array}{l}15.0 \\
\pm 5 \\
(29)\end{array}$ \\
\hline & $\begin{array}{l}\text { Subjects without } \\
\text { latent diabetes }\end{array}$ & $\begin{array}{r}33 \\
\pm 10\end{array}$ & $\begin{array}{l}112 \\
\pm \\
\pm\end{array}$ & $\begin{array}{r}35 \quad 528 \\
\pm 161\end{array}$ & $\begin{array}{r}467 \\
\pm 161\end{array}$ & $\begin{array}{r}389 \\
\pm 171\end{array}$ & $\begin{array}{r}325 \\
+166\end{array}$ & $\begin{array}{r}306 \\
\pm 169\end{array}$ & $\begin{array}{r}44 \\
\pm 20\end{array}$ & $\begin{array}{r}259 \\
\pm 83 \\
(14)\end{array}$ & $\begin{array}{c}1.4 \\
\pm 1.4 \\
(9)\end{array}$ & $\begin{array}{l}15.1 \\
\pm 7 \\
(14)\end{array}$ \\
\hline & $\begin{array}{l}\text { Subjects with } \\
\text { latent diabetes }\end{array}$ & $\begin{array}{r}45 \\
\pm 11\end{array}$ & $\begin{array}{r}127 \\
\pm \quad 22\end{array}$ & $\begin{array}{rr}21 \quad 605 \\
\pm 154\end{array}$ & $\begin{array}{r}564 \\
\pm 154\end{array}$ & $\begin{array}{r}488 \\
\pm 130\end{array}$ & $\begin{array}{r}418 \\
+122\end{array}$ & $\begin{array}{r}392 \\
\pm 143\end{array}$ & $\begin{array}{r}36 \\
\pm 17\end{array}$ & $\begin{array}{c}235 \\
\pm 46 \\
(15)\end{array}$ & $\begin{aligned} & 0.9 \\
+ & 0.6 \\
& (7)\end{aligned}$ & $\begin{array}{l}14.5 \\
+4 \\
(15)\end{array}$ \\
\hline & $\begin{array}{l}\text { Normal weight } \\
\text { prediabetics }\end{array}$ & $\begin{array}{r}31 \\
\pm 11\end{array}$ & $\begin{array}{r}93 \\
+\quad 8\end{array}$ & $\begin{array}{r}519 \\
\pm 190\end{array}$ & $\begin{array}{r}458 \\
\pm 151\end{array}$ & $\begin{array}{r}393 \\
\pm 178\end{array}$ & $\begin{array}{r}335 \\
\pm 171\end{array}$ & $\begin{array}{r}318 \\
\pm 178\end{array}$ & $\begin{array}{r}43 \\
+21\end{array}$ & $\begin{array}{r}243 \\
\pm 64 \\
\quad(7)\end{array}$ & $\begin{array}{r}1.4 \\
+1.8 \\
(5)\end{array}$ & $\begin{array}{l}11.5 \\
+8 \\
(7)\end{array}$ \\
\hline 3 & $\begin{array}{l}\text { Overweight } \\
\text { prediabetics } \\
(>10 \%)\end{array}$ & $\begin{array}{r}35 \\
\pm \quad 9\end{array}$ & $\begin{array}{r}130 \\
\pm \quad 20\end{array}$ & $\begin{array}{r}18538 \\
\pm 167\end{array}$ & $\begin{array}{r}474 \\
\pm 174\end{array}$ & $\begin{array}{r}386 \\
\pm 167\end{array}$ & $\begin{array}{r}314 \\
+167\end{array}$ & $\begin{array}{r}295 \\
\pm 164\end{array}$ & $\begin{array}{r}46 \\
\pm 17\end{array}$ & $\begin{array}{c}275 \\
\pm 102 \\
\quad(7)\end{array}$ & $\begin{array}{r}1.4 \\
\pm 0.9 \\
(4)\end{array}$ & $\begin{array}{c}18.6 \\
\pm 5 \\
(7)\end{array}$ \\
\hline & $\begin{array}{l}\text { Control group } \\
\text { (normal weight) }\end{array}$ & $\begin{array}{r}33 \\
\pm 12\end{array}$ & $\begin{array}{r}98 \\
\pm \quad 9\end{array}$ & $\begin{array}{rr}28 & 485 \\
& \pm 124\end{array}$ & $\begin{array}{r}402 \\
\pm 133\end{array}$ & $\begin{array}{r}300 \\
\pm 130\end{array}$ & $\begin{array}{r}237 \\
\pm 130\end{array}$ & $\begin{array}{r}215 \\
\pm 126\end{array}$ & $\begin{array}{r}57 \\
\pm 18\end{array}$ & $\begin{array}{c}223 \\
\pm 33 \\
(13)\end{array}$ & $\begin{array}{c}0.9 \\
+0.55 \\
(9)\end{array}$ & $\begin{array}{l}13.0 \\
\pm 2.3 \\
(13)\end{array}$ \\
\hline
\end{tabular}

number in brackets $=n$ 


\section{Literatur}

1. Bagdade, J.D., E.L. Brenman, and D. Porte: The significance of basal insulin levels in the evaluation of the insulin response to glucose in diabetic and nondiabetic subjects. J. clin. Invest. 46, 1549-1557 (1967).

2. Bottermann, P., K. Sohwarz u. K. Kopetz: Über das Verhalten der insulinähnlichen Aktivität im Serum bei der Fettsucht. Dtsch. med. Wschr. 90, 917-921 (1965).

3. Camerini-Dávalos, R.A.: Biochemical and histological aspects of prediabetes. In: LEIBEL, B.S., and G.A. WRENSHALL Eds: On the nature and treatment of diabetes. pp. 657-668. Amsterdam: Excerpta med. 1965.

4. Cerast, E., and R. LUFT: Insulin response to glucose infusion in diabetic and non-diabetic monozygotic twin pairs. Genetic control of insulin response? Acta endoer. (Kbh.) 55, 330-345 (1967).

5. - - "What is inherited - what is added"; Hypothesis for the pathogenesis of diabetes mellitus. Diabetes 16, 615-627 (1967).

6. Colwere, J.A., and A. LeIN: Diminished insulin response to hyperglycemia in prediabetes and diabetes. Diabetes 16, 560-565 (1967).

7. Conard, V., I.R.M. Franckson, P.A. Bastennte, J. Kmstens, and L. Kovacs: Etude critique du triangle d'hyperglycémie intraveineux chex l'homme normal et détermination d'un "coefficient d'assimilation glucidiques. Arch. int. Pharmacodyn. 13, 277-292 (1953).

8. DAwEKE, H.: Bestimmung der insulinähnlichen Aktivität im Blut des Menschen unter normalen und pathologischen Bedingungen, p. 65-68. Habilitationsschrift, Universität Düsseldorf 1964.

9. - H. van LANDEgHeM, I. BaCH, H. ZIMTMERMANN $u$. A. BREITBACH: Bestimmung der insulinähnlichen Alktivität und der physiologischen Insulinreserve bei schwerer Adipositas. Klin. Wschr. 43, 185-190 (1965).

10. DoLe, V.P.: A relation between non-esterified fatty acids in plasma and the metabolism of glucose. $J$. clin. Invest. 35, 150-154 (1956).

11. GRAdY, H.J., and M.A. LAMAR: Glucose determination by automatic chemical analysis. Clin. Chem. 5, $542-550(1959)$.

12. Grodsky, G.M., J.H. Karam, F.CH. PavLatos, and P.H. Forsham: Serum-insulin response to glucose in prediabetic subjects. Lancet $1965 \mathrm{I}, 290-291$.

13. Hales, C.N., and P.J. Ravole: Immunoassay of insulin with insulin-antibody precipitate. Biochem. $J$. $88,137-146$ (1963).

14. HofHMANN, W.S.: A rapid photoelectric method for the determination of glucose in blood and urine. J. biol. Chem. 120, $51-55(1937)$.

15. JAHNKe, K., H. DaWEKe, W. SchIlling, R. RÜ̈nAUVER u. K. OBERDIsse: Der potentielle Diabetes (sog. Praediabetes), Verh. dtsch. Ges. inn. Med. 72. Kongr. 1966, p. 851-868.

16. Karam, J.H., G.M. Grodsky, and P.H. Forsham: Excessive insulin response to glucose in obese subjects as measured by immunochemical assay. Diabetes 12, $197-204$ (1963).

17. Kreisberg, R.A., B.R. Bosheri, J. Dirtactoo, and R.F. Roddam: Insulin secretion in obesity. New Engl. J. Med. 276, 314-319 (1967).

18. KRFUTz, F.H.: Enzymatic determination of glycerol in the measurement of triglycerides. Int. Congr. for Clin. Chem., Detroit 1963, (personal communication.)
19. Lochner, W., u. M. NAsSERI: Weitere Untersuchungen über den Eigenstoffwechsel der Lunge, insbesondere die Freisetzung nichtveresterter Fettsäuren. Pflügers Arch. ges. Physiol. 272, 180 (1960).

20. Martin, D.B., A.E. Renold, and Y.M. Dagenais: An assay for insulin like activity using rat adjpose tissue. Lancet $1958 \mathrm{II}, 76-77$.

21. Mmiani, F., J. Laweckt, K.M. Bartelt u. E.F. PFEIFFER: Immunologisch meBbares Insulin (IMI) bei Stoffwechselgesunden, Fettsüchtigen und adipösen Diabetikern nach intravenöser Gabe von Glucose, Tolbutamid und Glukagon. Diabetologia 3, $422-426$ (1967).

22. Oberdisse, K., K. JahNkE u. H. Daweke: InsulinAntagonisten, Insulin-Antikörper, Insulin-Resistenz, Beziehungen zwischen Fett- und Kohlenhydratstoffwechsel. Die Erfassung praediabetischer Zustände. Gastroenterologia, Suppl. ad Vol. 104, 18-45, 1965.

23. Perciey, M., and D.M. KIPNIs: Plasma insulin responses to glucose and tolbutamide of normal weight and obese diabetic and nondiabetic subjects. Diabetes 15, $867-874$ (1966).

24. PxKe, D.A., and K.W. Taylor: Glucose tolerance and serum insulin in unaffected identical twins of diabetics. Brit. med. J. 1967 4, 21-22.

25. Rabinowitz, D., and K.L. ZinRLer: Forearm metabolism in obesity and its response to intraarterial insulin. Characterization of insulin resistance and evidence for adaptive hyperinsulinism. J. elin. Invest. 41, $2173-2181$ (1962).

26. RIOKETTS, H.T., R.A. ChERRY, and L. KIRITEINS: Biochemical studies of "prediabetes". Diabetes 15, $880-888$ (1966).

27. Rosenthal, H.L., M.M. Pfuuke, and J. Callerami: The colorimetric estimation of serum fatty esters. Clin. chim. Acta 4, 329 (1959).

28. Rünnauver, R., J. HerRmain u. H. Daweikw: Der unspezifische Einfluß eines Serumfaktors auf die radioimmunologische Insulinbestimmungsmethode nach Hales und Randle. Klin. Wschr. 44, 471-473 (1966).

29. Samaan, N., J. Brown, R. Fraser, and I. Trayner: Effect of obesity and of starvation on insulin activity. Brit. med. J. 1965 I, $1153-1156$.

30. Schilling, W.H., K. Oberdissa, K.A. HÜter u. H. BLANK: Vergleichende Untersuchungen mit der oralen und intravenösen Glucosebelastung zur Erfassung einer verminderten Kohlenhydrattoleranz. Diabetologia 1, 187-194 (1965).

31. SeLTZER, H.S., and W.L. SMrrTH: Plasma insulin activity after glucose. An index of insulinogenic reserve in normal and diabetic men. Diabetes 8, 417-424 (1959).

32. ShImA, K., and P.P. FOA: Serum immunoreactive insulin (TRI) and free fatty acids (FFA) responses to glucose and tolbutamide in diabetic, potentially diabetic and normal subjects. Excerpta med., Int. Congx. Series No. 140, 1967, p. 61-62.

33. Somidner, J.S., R.F. Wilutams, M.J. Garcta, R.E. Glesason, and A. MARBLe: Evidence for a normal response of serum immunoreactive insulin (IRI) to an intravenous glucose tolerance test (IVGTT) in prediabetic males. Diabetes 15, 518 (1966).

34. Strinke, J., R. Camerint, A. Marble, and A.E. RENoLD: Elevated levels of serum insulin-like activity (ILA) as measured with adipose tissue in early untreated diabetes and prediabetes. Metabolism 10, $707-711$ (1961).

35. - J.S. Somldner, R.A. CaMgrint-DÁvalos, and A. F. Rmnoud : Studies on serum insulin-lilke activity (ILA) in prediabetes and early overt diabetes. Diabetes 12, $502-507$ (1963). 
36. Trout, D.L., E.H. Estes, jr., and S.J. Frtedberg: Titration of free fatty acids of plasma: a study of current method and a new modification. J. Lipid Res. 1, 199 (1959/1960).

37. UNGER, R.H.: Diskussionsbemerkung zu dem Vortrag: E.F. Pletrfers: Recognized diabetogenic hormones and diabetes in men. In: LeIBEL, B.S., G.A. WrENSHALT, Eds: On the nature and treatment of diabetes, p. 390. Amsterdam: Excerpta med. 1965.

38. WILKERSON, H.L.: The effect of prior carbohydrate intake on the oral glucose tolerance test. Diabetes $\mathbf{9}$, $386-391$ (1960).
39. YALOW, R.S., S.M. GLToK, J. Roth, and S.A. BgrsoN: Plasma insulin and growth hormone levels in obesity and diabetes. Ann. N. Y. Acad. Sci. 131, $357-373$ (1965).

40. ZIATKIS, A., B. Z ${ }_{\text {AK, }}$ and A.J. BoxLE: A new method for the direct determination of serum cholesterol. J. Lab. clin. Med. 41, 486-492 (1953).

Doz. Dr. H. DaweikH 2. Med. Klinik und Poliklinik der Universität Düsseldorf 4 Düsseldorf Moorenstr. 5 